\title{
SUNSHINE: Smart UrbaN ServIces for Higher eNergy Efficiency
}

\author{
Manfred SCHRENK ${ }^{1}$, Wolfgang W. WASSERBURGER ${ }^{1}$, \\ Barbara MUŠIC $\check{C}^{2}$ and Linda DÖRRZAPF ${ }^{1}$ \\ ${ }^{1}$ CEIT ALANOVA, Schwechat/Austria·office@ceit.at \\ ${ }^{2}$ UIRS-Urban Planning Institute of the Republic of Slovenia, Ljubljana/Slovenia
}

This contribution was double-blind reviewed as full paper.

\begin{abstract}
SUNSHINE - Smart UrbaN ServIces for Higher eNergy Efficiency delivers innovative digital services, interoperable with existing geographic web-service infrastructures, supporting improved energy efficiency at the urban and building level. Specifically, SUNSHINE delivers a smart service platform accessible from both a web-based client and from an App for smartphones and tablets. In parallel to the technical integration a key standardization activity will also result in the definition of the extension of existing data model (CityGML), something very important for the pilots within SUNSHINE and its large scale uptake beyond current locations.

SUNSHINE is a European funded project in the competiveness and innovation framework program for the duration of three years with 16 partners from ten countries and it started in January 2013.
\end{abstract}

\section{Introduction}

Energy consumption levels in urban areas are increasing rapidly and so do global greenhouse gas emissions. Cities account for approximately two-thirds of global primary energy consumption and $71 \%$ of energy-related greenhouse gas emissions (IEA 2010). Energy demand patterns vary widely from city to city and across countries. Today, energy use in residential, commercial and public buildings accounts for $40 \%$ of total global final energy consumption (UNEP 2007). There is a huge energy saving potential in this sector and significant scope for adopting more efficient technologies and services in buildings (IEA 2010).

Energy certification of buildings is a key policy instrument for reducing the energy consumption and improving the energy performance of new and existing buildings. It should provide information that may increase demand for more efficient buildings, thereby helping to improve the energy efficiency of the building stock in urban areas (ARKESTEIJN \& VAN DIJK 2010).

SUNSHINE - Smart UrbaN ServIces for Higher eNergy Efficiency is a step towards this policy and is a tool to improve the energy efficiency of buildings and urban areas. This paper presents the project SUNSHINE funded by European Union under CIP framework,

Jekel, T., Car, A., Strobl, J. \& Griesebner, G. (Eds.) (2013): GI_Forum 2013. Creating the GISociety. (C) Herbert Wichmann Verlag, VDE VERLAG GMBH, Berlin/Offenbach. ISBN 978-3-87907-532-4.

(C) ÖAW Verlag, Wien. eISBN 978-3-7001-7438-7, doi:10.1553/giscience2013s18. 
which will develop a smart service platform for planners and public administration to extract analytical indicators necessary for the definition of energy saving policies for the existing public buildings asset and to define energy pre-certification mechanisms. Further, building managers can use SUNSHINE to optimize energy use of large-scale optimization of public illumination network. SUNSHINE delivers a smart service platform accessible from both a web-based client and from an app for smartphones and tablets. An important aspect of the SUNSHINE project is the use of a scalable approach, based on CityGML standard format, which makes a simultaneous representation of the energy balance at the urban scale. CityGML is an open data model and XML-based format for the storage and exchange of virtual 3D city models particularly suited to GIS applications (DALLA COSTA et al. 2011). The SUNSHINE technology will be the result of the customization and integration of existing software components developed by other EC-funded projects focusing on smart-city technologies, including BRISEIDE, i-SCOPE and i-Tour (DE AMICIS et al. 2010).

\section{SUNSHINE Scenarios - Use Cases and Technical Solution}

Within the SUNSHINE project three different scenarios are planned to deliver web-services compliant with Open Geospatial Consortium (OGC). In particular, the SUNSHINE platform will allow three following scenarios.

\subsection{Scenario 1: Assessment of energy performances and electronic energy pre-certification}

This scenario will allow automatic large-scale assessment of building energy behavior based on data available from public services (e.g. cadaster, planning data etc.), which have been granted as described within the letter of commitment. The information on energy performances will be used to automatically create urban-scale "ecomaps" to be used for planning activities and large-scale energy pre-certification purposes.

\subsubsection{Use case of scenario 1}

A building manager from the local public housing agency of Ferrara, Italy, starts a webclient to assess energy behavior of a set of 150 public buildings located city-wide (note that a single public building asset may be a multi-unit dwelling such as an apartment block).

The web-client connects to a SUNSHINE smart service that in turn connects to existing web services run by several departments of the municipality (e.g. cadastre, urban planning department etc.). The latter expose relevant data (including type of building, use category, geometry, size and shape, age and climatic zone, etc.) that has been compiled to ensure compliancy to a single interoperable profile of standards from OGC.

The SUNSHINE smart service uses this information to generate the $3 \mathrm{D}$ model of the relevant portion the city encoded as CityGML standard format, including estimation on performances for each building according to the SUNSHINE CityGML ADE (Application Domain Extension) on building energy efficiency. The information calculated by the sys- 
tem can also be double checked against actual consumption values available from the local energy provider.

As result the system returns both a $2 \mathrm{D}$ and 3D "ecomaps" (the latter being the full CityGML model), whereby each public building is classified according to their theoretical energy performance.

\subsubsection{Proposed technical solution}

SUNSHINE will leverage on existing OWS (OGC Web Services) available from public administrations such as cadaster, urban planning department, etc., implemented EU-wide in line with the INSPIRE "INfrastructure for SPatial InfoRmation in Europe" Directive (2007/2/EC, 2007).

Data available from the aforementioned OWS is exposed over the web via standard protocols such as WFS (Web Feature Service), WCS (Web Coverage Service) or WMS (Web Map Service) either directly from the public administration of the city where the pilot is located or on the project infrastructure.

\subsection{Scenario 2: Heating and cooling forecast and alerts}

This scenario particularly addresses existing buildings that can provide the biggest energy performance gains with the simplest activities.

The previous assessment will be used, together with localized weather forecasts available through interoperable web-services, to ensure optimization of energy consumption of heating and cooling systems through automatic alerts that will be sent via SUNSHINE App.

\subsubsection{Use case of scenario 2}

On January 2013, in the airport city Schwechat located next to Vienna, high-energy consumption was reported in the previous few weeks due to cold weather. Weather forecasts then predicted a sharp and significant increase in daily temperatures from minus ten degrees to around plus eight degrees. The building manager of the "Multiversum", a multipurpose event hall in Schwechat (fugure 1), had previously installed the SUNSHINE App on his

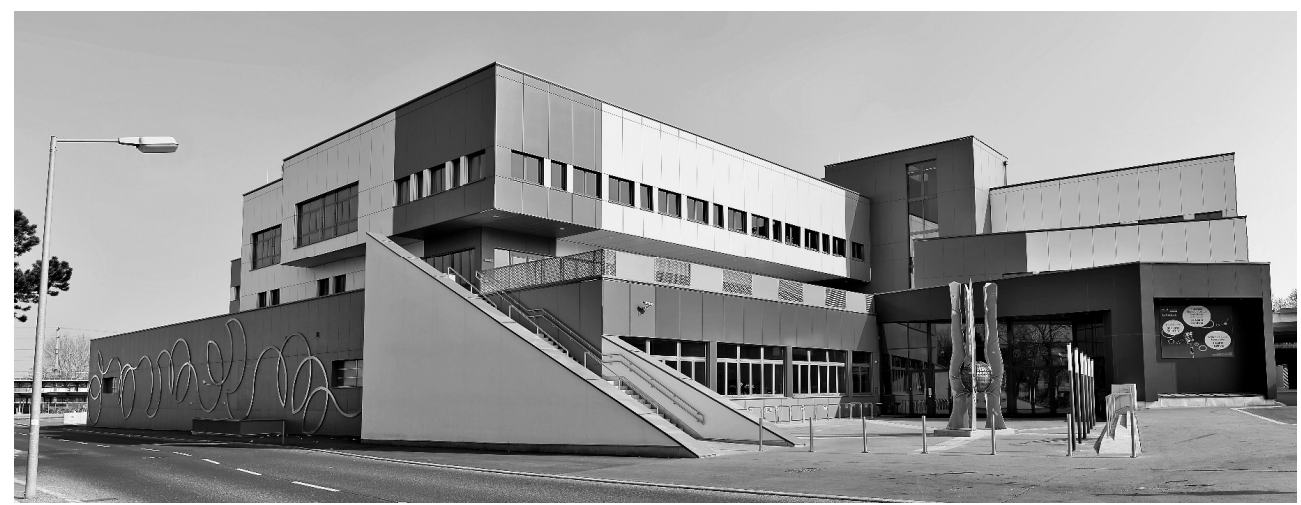

Fig. 1: The multipurpose hall "Multiversum" in Schwechat (Source: Multiversum) 
tablet. The App was configured with information on the type of heating system and position of the "Multiversum". The building manager had set an alert that would inform him of extreme and unseasonable changes in weather conditions. He receives detailed information on the dynamic rate scheme for the following days which significantly decreases the costs for energy consumptions. This gives him the possibility to react immediately and to adjust the heating.

The SUNSHINE system receives the weather forecasts predicting the sharp increase in temperature. The system retrieves the list of buildings according to their energy performances and it starts sending notifications, according to the estimated thermal inertia of each single building, warning to turn off heating system, due to improving weather condition, at a given time of the day to ensure an optimally comfortable transition to higher external temperatures. The same scenario could be applied both in hot summer days (when air conditioning is at its peak) and during cold winter days to reduce concentration of PM10 due to excessive heating of buildings.

\subsubsection{Proposed technical solution}

SUNSHINE will leverage on existing weather services that will be customized to provide access via interoperable standards from the SWE (Sensor Web Enablement) family of standards from OGC. This way the SUNSHINE web service will integrate existing weather services to provide both past and forecast weather data specific for each building.

A smart web service (figure 2) will use existing CityGML model of the urban environment to issue, based on weather data, alerts based on energy behaviors of building. The service

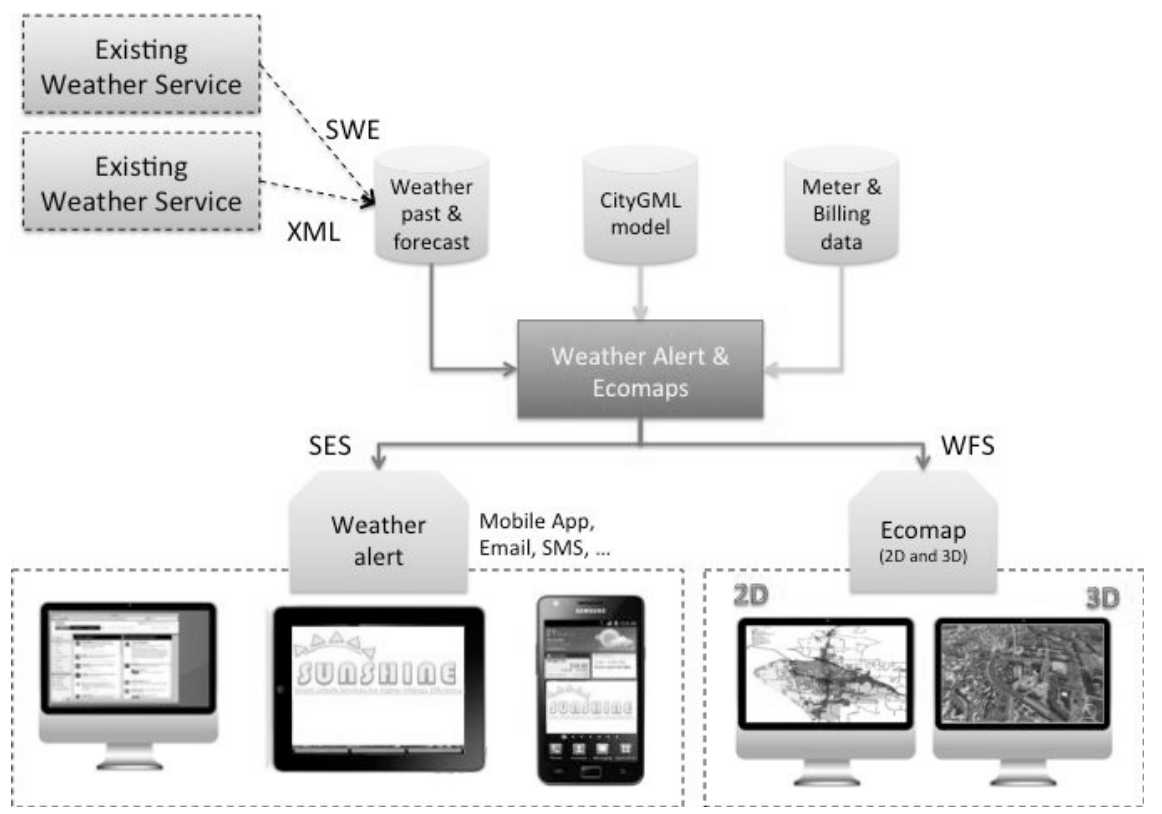

Fig. 2: The data flow within the scenario 2 (dashed blocks denote existing technology) 
will be based on a mathematical model. Meter and billing data will be retrieved via the utility services using the IEC messaging standards (IEC 61968-9) and the IEC Web Services implementation profiles (IEC 61968-100).

The results of the service will be an "ecomap" of predicted building energy response according to changing weather conditions. The ecomaps will be available, as web service, through the OGC standard WFS (Web Feature Service).

\subsection{Scenario 3: Optimization of power consumption of public lighting systems}

SUNSHINE will ensure interoperable control of public illumination systems based on Automatic Meter Reading (AMR) facilities remotely accessible, via interoperable standards, from a web-based client as well as from an App for smartphones or tablets.

\subsubsection{Use case of scenario 3}

An operator of the Municipality of Bassano del Grappa (Italy) wants to optimize illumination (indoor and outdoor) levels of public buildings (e.g. City Council) and building of public interest (e.g. stadium). He starts a 3D web client that shows the map of the city and the public illumination network. The web client operates as a dashboard from which he can control, through an interoperable standard, real time status of public illumination system. This allows him to check, from a single point of access, the functioning of the various parameters of the entire lighting system through an interactive mapping environment, identifying for instance damages, power losses or simply inefficient use (for instance lights being turned on when unnecessary).

The operator can control lighting conditions to avoid unnecessary illumination of common areas whenever not required. He sets the illumination level down by $50 \%$ in the evening when all activities inside the stadium are finished. Similar optimization is performed for other public buildings. The dimming (as well as other control) can be performed manually at the control panel level (for the entire line) or at a single illumination unit.

\subsubsection{Proposed technical solution}

SUNSHINE will integrate, through web services, an existing client with current remote unit messages and controls transforming messages to standard protocols from the OGC, within on the SWE (Sensor Web Enablement) standard family. In particular messages among different illumination units, typically exchanged as industry standards EN 50065-1, will be interconnected, at the central level, with an interface exposing the information as Sensor Observation Service (SOS), Sensor Alert Service (SAS) and Sensor Event Service (SES), the latter two being used to configure alerts.

Real time information on illumination network is accessed from both a desktop and a mobile client (for tablets or smartphones) that accesses, via standard OWS (OGC Web Services), information on the area (maps of buildings, road network, lighting network) on top of which information on real-time status of the system is rendered according to SOS standard. 
The advantage of this approach is that any compatible client (both mobile and desktop) will be able to retrieve real-time information on specific illumination units in a standardized protocol according to their specific location in space. The mobile client can also be used for onsite surveying to georeferenced components of outdoor illumination network within the mapping environment.

\section{Pilots within SUNSHINE}

The three described scenarios will be piloted in the context of nine sites across five countries that include, e.g. for Italy 20 public buildings in Ferrara; 60 technical buildings across the Trentino Province; 4 public illumination lines in the center of Bassano del Grappa (Italy), 5 public illumination lines in the city of Rovereto (Italy) 3 building complexes in the area of Val di Non (Italy) and their outdoor public illumination systems.

For Austria, it will be the multipurpose hall "Multiversum" in Schwechat, Austria. It is a complex opened in 2011 and used for sport and cultural events, but also for exhibitions, fairs, congresses, conventions and business events. Other pilots are located in Croatia, Greece and Malta.

SUNSHINE will be piloted for a duration of 12 months and it will target at energy and emission savings ranging, within the various pilots, from $10 \%$ to $30 \%$, with higher savings being foreseen for pilots relying on older buildings, or equipped with older heating, cooling or lighting technologies. Energy savings will be compared to a one-year baseline data acquired, during the first stages of the project prior to the deployment of the pilots.

\section{Conclusion and Future Work}

SUNSHINE is an EU project that aims to improve energy efficiency of public buildings. In parallel to technical integration, a key standardization activity will also result in the extension of the existing data model (CityGML) through a specific Application Domain Extension (ADE) on building energy behavior. Local administrations play an important role within the project and are constantly involved in the process.

The first step within the project will be to precisely define the use cases to be addressed by the various pilots and collect requirements for the deployment of the SUNSHINE system, including, e.g. user, training, service requirements and a definition of the software architecture of the whole SUNSHINE system. Further steps for the SUNSHINE implementation are the identification, collection and harmonization of all available information to support the SUNSHINE pilots.

\section{Acknowledge}

SUNSHINE is a project funded under the ICT Policy Support Programme of EU as a part of the Competitiveness and Innovation framework Programme (CIP). It will be realized with an international multi-disciplinary consortium including the following partners: 
Fondazione Graphitech (leadpartner) (Italy), Gistandards LTD (UK), SinerGIS SRL (Italy), Cadzow Communications Consulting Limited (UK), Zancarli Ivo - Grafica Light Di Zancarli Ing Ivo (Italy), Informatica Trentina SpA (Italy), Epsilon International SA (Greece), Fundación ESADE (Spain), CEIT Alanova Gemeinnutzige GMBH (Austria), Trentino Network Srl (Italy), HEPESCO (Croatia), Farisa Asesores Y Consultores S.L (Spain), Urbasofia SRL (Romania), Urban Planning Institute of the Republic of Slovenia (Slovenia), GeoSYS Limited (Malta) and SET Distribuzione Spa (Italy).

\section{References}

ARkesteiJn, K. \& VAN DiJK, D. (2010), Energy Performance Certification for New and Existing Buildings, EC Cense P156, EC, available at: www.iee-cense.eu.

Dalla Costa, S., Roccatello, E. \& Rumor, M. (2011), A CITYGML 3D geodatabase for buildings' energy efficiency, International Archives of the Photogrammetry, Remote Sensing and Spatial Information Sciences, 19-24.

De Amicis, R., Conti, G. \& PRANDI, F. (2010), An Integrated Framework For SpatioTemporal Data Management: The Project BRISEIDE - BRIdging SErvices Information and Data for Europe. In: WebMGS 2010 - 1st International Workshop on Pervasive Web Mapping, Geoprocessing and Services. Como, Italy.

EUROPEAN PARLIAMENT AND COUNCIL (2007), Directive 2007/2/EC of 14 March 2007, Infrastructure for Spatial Information in the European Community (INSPIRE).

IEA (2010), Energy Performance Certification of Buildings - A policy tool to improve energy efficiency, Paris, France. www.iea.org.

IEC: 61968-9 (2009), Application integration at electric utilities - System interfaces for distribution management - Part 9: Interfaces for meter reading and control.

IEC: 61968-100 (2011), Application integration at electric utilities - System interfaces for distribution - Part 100: Implementation Profiles for IEC.

UNEP (2007), Buildings and climate change - Status, Challenges and Opportunities, Kenia. www.unep.org. 